\title{
Nonselfadjoint Operators in Diffraction and Scattering*)
}

\author{
A. G. Ramm, Ann Arbor, MI \\ Communicated by R. P. Gilbert
}

\section{Contents}

1. Introduction

2. When do the eigenvectors of $T$ and $A$ form a basis of $H$ ?

3. When do $T$ and $A$ have no root vectors?

4. What can be said about the location and properties of the complex poles?

5. How to calculate the poles of the Green function?

Do the poles depend continuously on the boundary of the obstacle?

Appendix 1. Losses in open resonators

Appendix 2. An example on complex scaling

Appendix 3. Variational principles for eigenvalues of compact nonselfadjoint operators

Bibliographical note

Unsolved problems

References

\section{$\$ 1$ Introduction}

Consider the following problem

(1) $\left(\triangle+k^{2}\right) u=0$ in $\Omega$,

(2) $\quad \partial u \mid \partial N=f$ on $\Gamma$,

(3) $|x|(\partial u / \partial|x|-\mathrm{i} k u) \rightarrow 0$ as $|x| \rightarrow \infty$,

where $\Omega$ is an unbounded domain with a smooth closed compact surface $\Gamma$, $\Gamma \in C^{2}$.

If we look for a solution in the form

(4)

$$
u=\int_{\Gamma} \frac{\exp \left(\mathrm{i} k r_{x y}\right)}{4 \pi r_{x y}} g(y) \mathrm{d} y, r_{x y}=|x-y|,
$$

then

(5) $g=A g-2 f$,

*) This work was supported by AFOSR F4962079 C0128. 
where

$$
A g=\int_{\Gamma} \frac{\partial}{\partial N_{t}} \frac{\exp \left(\mathrm{i} k r_{t y}\right)}{2 \pi r_{t y}} g(y) \mathrm{d} y .
$$

If the boundary condition is of the form

$$
u=f \text { on } \Gamma \text {, }
$$

then the integral equation for $g$ takes the form

$$
T g=f,
$$

where

$$
T g=\int_{\Gamma} \frac{\exp \left(\mathrm{i} k r_{t y}\right)}{4 \pi r_{t y}} g(y) \mathrm{d} y .
$$

If one wishes to solve equations (8), (5) by means of expansions in root vectors, one must prove that the root vectors of operators $A$ and $T$ form a basis of $H=L^{2}(\Gamma)$. Both operators are compact and nonselfadjoint. Apriori it is not clear why these operators have eigenvectors: e.g. Volterra operator has no eigenvectors. In applications it is more convenient to use only eigenvectors, because calculations with the root vectors are more complicated. This leads to the following question: when does a nonselfadjoint operator have no root vectors? Here and below we use the word root vectors meaning associated root vectors. The definition is: if $A g=\lambda g, g \neq 0$, then $g$ is an eigenvector; if equation $A h_{1}-\lambda h_{1}=g$ is solvable, then $h_{1}$ is an associated vector (or root vector); the set $\left(g, h_{1}, \ldots h_{s}\right)$ is called a Jordan chain with the length $s+1$, if $(A-\lambda) g=0$, $(A-\lambda) h_{1}=g,(A-\lambda) h_{k}=h_{k-1}, 2 \leqslant k \leqslant s$, vectors $h_{1}, \ldots h_{s}$ are called root vectors. An isolated eigenvalue $\lambda$ is called a normal eigenvalue if its algebraic multiplicity is finite and the Hilbert space $H$ can be decomposed into the direct sum of subspaces $H=L_{\lambda} \dot{+} R_{\lambda}$, where $L_{\lambda}$ is the root subspace of $A$ and $R_{\lambda}$ is an invariant subspace for $A$ in which $(A-\lambda I)^{-1}$ exists. The root subspace $L_{\lambda}$ is the linear span of all eigen and root vectors of $A$ corresponding $\lambda$. It is well known that $\lambda$ is a normal eigenvalue iff the projector $P=-(2 \pi \mathrm{i})^{-1} \int_{|z-\lambda|=\varepsilon}(A-z I)^{-1} \mathrm{~d} z$ is finite-dimensional [1]. If $\lambda$ is a normal eigenvalue of $A$ then $\left(A-z D^{-1} \equiv R_{z}\right.$ has a simple pole at $\lambda$ iff the length of the Jordan chain is equal to 1 . It means that the eigensubspace of $A$ corresponding to $\lambda$ coincides with the root subspace of $A$ corresponding to $\lambda$. From the definition given above it follows that the pole $\lambda$ is simple iff $(A-\lambda)^{2} f=0 \Rightarrow(A-\lambda I) f=0$. In physical literature there is a great interest in equations of type (6), (8) and in their counterparts in the electromagnetic wave scattering theory [2]. Engineers used the singularity and eigenmode expansion methods for solution of exterior boundary value problems [3], [4]. What they call eigenmode expansion method (EEM) is actually an old Picard's method for solution of selfadjoint integral equations of the first kind. They suppose that the operator $T$ defined by (9) has eigenvectors.

$$
T f_{j}=\lambda_{j} f_{j}, \quad j=1,2, \ldots,\left|\lambda_{1}\right| \geqslant\left|\lambda_{2}\right|>\ldots,
$$


has not root vectors and the set of his eigenvectors $\left\{f_{j}\right\}$ forms a Riesz basis of $H=L^{2}(\Gamma)$. We remind the reader that $\left\{f_{j}\right\}$ is a Riesz basis of $H$ (or basis equivalent to an orthonormal basis $\left\{h_{j}\right\}$ of $H$ ) if a bounded invertible linear operator $B$ exists such that $B h_{j}=f_{j}$. We call an operator $B$ invertible if $B^{-1}$ is bounded and defined on $H$. Under such an assumption engineers solve equation (8) using the formula

$$
g=\sum_{j=1}^{\infty} \lambda_{j}^{-1}(k)\left(f, f_{j}\right) f_{j}
$$

The following questions are open and of interest to mathematicians:

1) when do the eigenvectors of $T$ and $A$ form a basis of $H$ ?

2) when there do not exist root vectors of $T$ and $A$ ?

These questions are far from trivial. In fact for the basic equation of the theory of lazers

$$
\int_{-1}^{1} \exp \left\{\mathrm{i}(x-y)^{2}\right\} f(y) \mathrm{d} y=\lambda f(x), \quad-1 \leqslant x \leqslant 1,
$$

nothing is known about the existence and properties of its eigen functions until now. Fortunately the situation is much better for the operators $A$ and $T$ and later we give some reasons for this statement.

The singularity expansion method (SEM) consists in the following. Given the nonstationary problem:

$$
\left\{\begin{array}{l}
u_{t t}=\Delta u, \quad t \geqslant 0, x \in \Omega \in \mathbf{R}^{3}, \\
\partial u / \partial N=0, \text { on } \Gamma \\
u(0, x)=0, \quad u_{t}(0, x)=f(x),
\end{array}\right.
$$

and assuming

$$
v(x, k)=\int_{0}^{\infty} \exp (\mathrm{i} k t) u(x, t) \mathrm{d} t
$$

we obtain:

$$
\left\{\begin{array}{l}
\Delta v+k^{2} v=-f, \quad \partial v / \partial N=0 \quad \text { on } \Gamma, \\
(\partial v / \partial|x|-i k v)=o\left(|x|^{-1}\right) .
\end{array}\right.
$$

If $G(x, y, k)$ is the Green function for this problem, then

$$
v=\int_{\Omega} G(x, y, k) f \mathrm{~d} y .
$$

From (14), (16) we obtain

$$
u(x, t)=\frac{1}{2 \pi} \int_{-\infty}^{\infty} v \exp (-\mathrm{i} k t) \mathrm{d} k .
$$

For the sake of simplicity we assume that $f \in C_{0}^{\infty}(\Omega)$. The function $G(x, y, k)$ can be continued analytically on the whole complex plane $k$. It is analytic in the upper half plane $\operatorname{Im} k \geqslant 0$ and meromorphic in the lower half-plane $\operatorname{Im} k<0$. For details see [5], [6]. Suppose that 


$$
|v| \leqslant \frac{C}{1+|k|^{a}}, \quad \operatorname{Im} k>-b, b>0, a>0.5 \text {. }
$$

Then we can move down the contour of integration in (17)

$$
u(x, t)=\frac{1}{2 \pi} \int_{-\mathrm{i} c-\infty}^{-\mathrm{i} c+\infty} v(x, k) \exp (-\mathrm{i} k t) \mathrm{d} k, \quad 0<c<b .
$$

From this it follows that

$$
u(x, t)=\exp (-c t) w(x, t), \quad 0<c<b,
$$

where

$$
w(x, t)=\frac{1}{2 \pi} \int_{-\infty}^{\infty} v(x,-\mathrm{i} c+y) \exp (-\mathrm{i} y t) \mathrm{d} y .
$$

From (18) it follows that $v \in L^{2}(\mathrm{R})$ and $w \in L^{2}(\mathrm{R})$. Suppose that the poles $k_{j}$ of $v(x, k)$ satisfy the inequality

$$
\operatorname{Im} k_{j}<-F\left(\left|\operatorname{Re} k_{j}\right|\right),
$$

where $F(x)$ is a continuous positive function,

$$
F(x)>0, \quad F(-x)=F(x), \quad F(x) \rightarrow+\infty \text { as } x \rightarrow \infty .
$$

If (18) holds in the domain

$$
\operatorname{Im} k>-F(|\operatorname{Re} k|),
$$

then by moving the contour of integration in (17) we get the asymptotic expansion (singularity expansion):

$$
u(x, t)=\sum_{j=1}^{n} \mathrm{e}^{-\mathrm{i} k_{j} t} v_{j}(x)+o\left(\mathrm{e}^{-\left|\mathrm{Im} k_{n}\right| t}\right)
$$

This leads to the following questions:

3) What can be said about location of the poles $k_{j}$ ? When does (18) hold? When does (18) hold in the domain (22)?

4) What can be said about the properties of the poles $\left\{k_{j}\right\}$ ? How to calculate these poles? Do these poles depend continuously on the boundary?

5) To what extent does the set of poles $\left\{k_{j}\right\}, \operatorname{Im} k_{j}<0$ determine the shape of the obstacle?

These questions are discussed in this paper. They are of interest in applications and difficult from the mathematical point of view.

All of the results concerning operators $A$ and $T$ can be obtained for the analogous integral operators in the electromagnetic wave scattering theory. In what follows formula (1.6) will denote formula (6) in $\S 1$. We use autonomous numeration throughout the sections. 


\section{$\S 2$ When Do the Eigenvectors of $T$ and $A$ Form a Basis of $H$ ?}

\section{Bases with Brackets. Tests for Completeness and Basisness}

Let $\left\{h_{j}\right\}$ be an orthonormal basis of $H, m_{1}<m_{2} \ldots$ a sequence of integers, $m_{l} \rightarrow \infty$ as $l \rightarrow \infty$, and let $H_{l}$ be the linear span of the vectors $h_{m_{l}}, h_{m_{l}+1}$, $\ldots h_{m_{l+1}-1}$. Let $\left\{f_{j}\right\}$ be a complete minimal system in $H$, and $F_{l}$ be the linear span of vectors $f_{m l}, \ldots f_{m_{l+1}-1}$. By basisness we mean the property of a system of vectors or subspaces to form a basis of $H$.

Definition 1 If a linear, bounded, invertible operator $B$ exists such that $B H_{l}=$ $F_{l}$ then the system $\left\{f_{j}\right\}$ is called a Riesz basis of $H$ with brackets (notation: $\left.\left\{f_{j}\right\} \in R_{b}(H)\right)$. If $m_{j}=j$ then $\left\{f_{j}\right\} \in R(H)$ and is called a Riesz basis of $H$.

Remark 1. It is known [1], that $\left\{f_{j}\right\} \in R_{b}(H)$ iff $C_{1}|f|^{2} \leqslant \sum_{l=0}^{\infty}\left|P_{L} f\right|^{2} \leqslant C_{2}|f|^{2}$, where $|\cdot|$ is the norm in $H, C_{2} \geqslant C_{1}>0$ are constants, $P_{l}$ is the projector on $F_{l}$, $f \in H$ is an arbitrary element of $H$. Projector $P_{l}$ is defined by the direct decomposition $H=F_{l}+G_{l}$, where $G_{l}$ is the union of the subspaces $F_{j}$ for $j \neq l$.

Definition 2 Denote by $Q_{l}$ the orthoprojector on $H_{l}$. If $\sum_{l=0}^{\infty}\left|P_{l}-Q_{l}\right|^{2}<\infty$ then the system $\left\{f_{j}\right\}$ is called a Bari basis with brackets (notation $\left\{f_{j}\right\} \in B_{b}(H)$ ).

Definition 3 A linear closed densely defined operator $L$ on a Hilbert space $H$ is called an operator with discrete spectrum iff its spectrum $\sigma(L)$ consists only of normal eigenvalues $\lambda_{j},\left|\lambda_{1}\right| \leqslant\left|\lambda_{2}\right| \leqslant \ldots\left|\lambda_{j}\right| \leqslant \ldots,\left|\lambda_{j}\right| \rightarrow \infty$ as $j \rightarrow \infty$.

Remark 2. If $L$ is a normal operator with discrete spectrum, $0 \notin \sigma(L)$, then $L^{-1}$ is conpact.

In what follows we assume for the sake of simplificity that $L$ is a selfadjoint operator with a discrete spectrum $\left\{\lambda_{j}\right\}, 0 \notin \sigma(L)$,

$$
\lambda_{j}=c j^{p}+0\left(j^{p_{1}}\right), \text { as } j \rightarrow \infty, p>0, c>0, p_{1}<p .
$$

Consider the operator

$$
A=L+Q
$$

where $Q$ is a (nonselfadjoint) linear operator,

$$
\left|L^{-a} Q f\right| \leqslant C_{a}|f|, \quad \forall f \in H, a<1, D(Q) \supset D(L) .
$$

Since

it is clear, that

$$
(L+Q-\lambda I)^{-1}=\left\{I+(L-\lambda I)^{-1} L^{a} L^{-a} Q\right\}^{-1}(L-\lambda I)^{-1}
$$

$$
\lambda \notin \sigma(A) \text { if }\left|(L-\lambda)^{-1} L^{a}\right|<C_{a}^{-1} .
$$

It is clear that

$$
\left|(L-\lambda I)^{-1} L^{a}\right| \leqslant \sup _{j}\left|\lambda_{j}-\lambda\right|^{-1}\left|\lambda_{j}\right|^{a} .
$$


If $\left|\lambda_{j}-\lambda\right| \geqslant\left|\lambda_{j}\right|^{a} C_{a} q$, where $q>1$ is arbitrary, then (5) holds. Hence we have proved the main part of the following lemma.

Lemma 1 Suppose that $L$ is a selfadjoint operator with a discrete spectrum, $Q$ is $a$ linear operator, $A=L+Q$, and (3) holds. Then $\sigma(A) \subset K$, where

$$
K=\bigcup_{j=1}^{\infty}\left\{\lambda\left|\lambda-\lambda_{j}\right|<\left|\lambda_{j}\right|^{a} C_{a} q, q>1\right\}
$$

and $\sigma(A)$ is discrete.

Proof. It remains to prove the last statement of Lemma 1. The statement follows immediately from the compactness of $(L-\lambda)^{-1}$ and boundedness of the operator $\left\{I+(L-\lambda)^{-1} L^{a} L^{-a} Q\right\}^{-1}$ in (4).

Remark 3. Estimates of type (6) were used earlier by Kacnelson [18], [3]. We made no use of assumption (1) so far.

We write $A \in R(H)$ if the root system of $A\left\{f_{j}\right\} \in R(H)$.

Theorem 1 ([18]) Under the assumptions (1), (3) $A \in R_{b}(H)$ if $p(1-a)=1$, and $A \in B_{b}(H)$ if $p(1-a)>1$.

Remark 4. We write $A \in R_{b}(H)\left(B_{b}(H)\right)$ if the root vectors of $A$ form a Riesz (Bari) basis of $H$ with brackets.

Remark 5. Actually for Theorem 1 to be true it is sufficient to use the following estimate instead of (1): $\lambda_{j} \geqslant c j^{p}$ (see [18]).

Remark 6. Under some additional assumptions M. S. Agranovich proved that the series in root vectors of $A$ converges rapidly (see Appendix in [3]).

Remark 7. Completeness of the root system of a linear operator $A$ in a Hilbert space $H$ can be proved by means of the following theorems.

Theorem 2 ([1]) If $L$ is a selfadjoint operator on a Hilbert space $H$ with a discrete spectrum, $0 \notin \sigma(L), Q$ is a linear operator $D(Q) \supset D(L), L^{-1} Q$ is compact and $p\left(L^{-1} Q L^{-1}\right)<\infty$, then the system of root vectors of $A=L+Q$ is complete in $H$. Remark 8 . The symbol $p(A)<\infty$ means that $A$ is compact and $\sum_{1}^{\infty} s_{n}^{p}<\infty$,
where $s_{n}=\lambda_{n}\left\{\left(A^{*} A\right)^{1 / 2}\right\}$ are the $s$-values of $A$.

Theorem 3 ([1]) The system of root vectors of a compact dissipative operator $A$ with nuclear imaginary component is complete in $H$ if $\lim _{n \rightarrow \infty} \inf n s_{n}(A)=0$.

Remark 9. A linear operator $A$ is called dissipative if $\operatorname{Im}(A f, f) \geqslant 0$ $\forall f \in D(A)$. A compact linear operator is called nuclear if $\sum_{1}^{\infty} s_{n}(A)<\infty$.

Theorem 4 ([11]) If $A \geqslant 0$ is compact, $B$ is dissipative and nuclear then the root system of $A+B$ is complete in $H$.

Theorem 5 ([43]) If (1), (3) hold $p_{1}<p-1$ and $p(1-a) \geqslant 2$ then $A \in R(H)$. 
Example 1 ([11]). Operator (1.9) can be split into the sum $T=T_{0}+T_{1}$, where $T_{0} g=\int_{\Gamma}\left(4 \pi r_{t y}\right)^{-1} g(y) \mathrm{d} y, T_{0}>0$, and $T_{1}=T-T_{0}$ is nuclear and dissipative. The last statements is easy to verify (see [11] for details). Thus from Theorem 4 it follows that the root system of operator (1.9) is complete in $H=L^{2}(\Gamma)$. Actually this system forms a Riesz basis as we shall prove later.

\section{Elliptic Pseudo-differential Operators (PDO) on $\Gamma$}

In order to explain how to prove that the root systems of operators $A$ (formula (1.6)) and $T$ (formula (1.9)) form a Riesz basis of $H$ we start with the operator $T$. It is clear that

$$
T=T_{0}+T_{1}
$$

where $T_{0}, T_{1}$ are defined in Example 1 . It is easy to verify that $T_{0}$ is an elliptic pseudo-differential operator on $\Gamma$ of order -1 and $T_{1}$ is a PDO of order $y<-1$ (in fact $y=-3$ ). Suppose that $\operatorname{Ker} T_{0}=\{0\}$. Then $L=T_{0}^{-1}$ exists, $L$ is a selfadjoint operator with discrete spectrum. If $\operatorname{Ker} T=\{0\}$, then

$$
\left(T_{0}+T_{1}\right)^{-1}=\left(I+L T_{1}\right)^{-1} L=L+Q,
$$

where $Q=-\left(I+L T_{1}\right)^{-1} L T_{1} L,\left|L^{-a} Q\right| \leqslant C$ for $a=2+\gamma<1$

because ord $L T_{1} L=2+\gamma<1$. Condition (1) is valid for PDO under very general assumptions [20]. Therefore one can apply Theorem 1 and obtain

Proposition 1 The root system of operator $T$ defined by formula (1.9) forms a Riesz basis of $H=L^{2}(\Gamma)$ with brackets.

Remark 10. It is easy to verify that $\operatorname{ker} T_{0}=\{0\} . \operatorname{ker} T=\{0\}$ if $\kappa^{2}$ is not an eigenvalue of the interior Dirichlet problem.

Remark 11. One can find e.g. in [21] how to calculate the order of an elliptic PDO.

Remark 12. It is possible (and in a way more reasonable) to choose $T_{0}=0.5$ $\left(T+T^{*}\right)$, because in this case $T_{1}$ will be of the order $-\infty$ for real $k>0$ since the kernel of $T_{1}$ is $\frac{\sin k r_{t y}}{r_{t y}} \in C^{\infty}$ (ord $T_{1}=-3$ for complex $\kappa$ ).

Consider now the operator $A$ defined by formula (1.6).

It is easy to verify that $A$ is a pseudo-differential elliptic operator, and ord $A=-1$. If $A_{0}=0.5\left(A+A^{*}\right), A_{1}=A-A_{0}$, then ord $A_{0}=-1$, ord $A_{1}$ $<-1$. If $\operatorname{ker} A_{0}=\{0\}$, and $\operatorname{ker} A=\{0\}$ one can use the arguments similar to ones used above and obtain the analogue of Proposition 1 for the operator $A$. If $\operatorname{ker} A_{0} \neq\{0\}$ then $\operatorname{dim} \operatorname{ker} A_{0}<\infty$ and $\operatorname{ker} A_{0} \subset C^{\infty}$. This statement follows from the apriori estimates for elliptic PDO [21]. Thus, one can add a finite dimensional operator $P$ to $A_{0}$ and subtract this operator from $A_{1}$. Since ker $A_{0} C$ $C^{\infty}$ operator $P$ can be chosen so that ord $\left(A_{0}+P\right)=\operatorname{ord} A_{0}=-1$ (ord $P=$ $-\infty)$, and $\operatorname{ker}\left(A_{0}+P\right)=\{0\}$. Hence, one can assume that ker $A_{0}=\{0\}$. If 
ker $A_{0}=\{0\}$ then $A_{0}^{-1}$ exists and has a discrete spectrum. Since ord $A_{1}<\operatorname{ord} A_{0}$ the operator $A_{0}^{-1} A_{1}$ is compact in $H$. From this argument and the formula $A=$ $A_{0}\left(I+A_{0}^{-1} A_{1}\right)$ it follows that the root subspace $N$ of $A$ corresponding to $\lambda=0$ is finite-dimensional. Therefore one can split $H$ into a direct sum $H=N+M$, where $N$ and $M$ are invariant subspaces of $A$ and $\left.\operatorname{ker} A\right|_{M}=\{0\},\left.A\right|_{M}$ denoting the restriction of $A$ to $M$. Hence, one can assume that $\operatorname{ker} A=\{0\}$. This completes the proof of the following proposition.

Proposition 2 The root system of operator $A$ defined by formula (1.6) forms a Riesz basis of $H$ with brackets.

\section{$\S 3$ When Do $T$ and $A$ Have no Root Vectors?}

1. A simple sufficient condition was given in [11]: in order that $T$ (or $A$ ) has no root vectors it is sufficient that $T$ is normal. This condition $T^{*} T=T T^{*}$ can be written explicitly [11] and it is a condition concerning the surface $\Gamma$. In [11] is was verified that for operator $T$ this condition is satisfied if $\Gamma$ is sphere. For linear antenna this condition is also satisfied [11]. Of course, this condition is not necessary. In a finite-dimensional Hilbert space $H$ every linear operator $A$ without root vectors is similar to a normal operator. Indeed, if $A$ has no root vectors then its eigenvectors $\left\{f_{j}\right\}$ form a basis of $H$. If $\left\{h_{j}\right\}$ is an orthonormal basis of $H, A f_{j}=\lambda_{j} f_{j}$ and $f_{j}=C h_{j}$, then $C^{-1} A C h_{j}=\lambda_{j} h_{j}$. It means that operator $C^{-1} A C$ is normal.

In infinite-dimensional Hilbert space $H$ this is not true: there exist compact operators whose eigenvectors span $H$ but these operators are not similar to a normal operator (an example is given in [24]).

2. In [23] the following observation was formulated: the eigensubspace and the root subspace of a compact operator $T$, corresponding to the number $\lambda$, coincide iff 1) $\lambda$ is a simple pole of the resolvent $(T-\lambda)^{-1}$, or iff 2) $(T-\lambda)^{2} f$ $=0 \Rightarrow(T-\lambda I) f=0$, or iff 3$)$ the operator $T-\lambda I$ does not have zeros in the subspace $R(T-\lambda I)$, where $R(A)$ denotes the range of $A$.

\section{$\$ 4$ What Can Be Said About the Location and Properties of the Complex Poles?} problem:

1. Consider the Green function $G(x, y, k)$ of the exterior Dirichlet

(1) $\left(\Delta+k^{2}\right) G=-\delta(x-y)$ in $\Omega$,

(2) $\left.G\right|_{\Gamma}=0$,

(3) $\quad|x|(\partial G / \partial|x|-\mathrm{i} k G) \rightarrow 0$ as $|x| \rightarrow \infty, k>0$.

Let $G_{0}=\left(4 \pi r_{x y}\right)^{-1} \exp \left(\mathrm{i} k r_{x y}\right)$. Then

$$
G(x, y, k)=G_{0}(x, y, k)-\int_{\Gamma} G_{0}(x, y, k) \mu(t, y, k) \mathrm{d} t, \quad \mu=\frac{\partial G}{\partial N_{t}},
$$


where $N$ is the unit of the outer normal to $\Gamma$ at the point $t$, and $\mu$ satisfies the equation

$$
\text { (5) } \mu+A \mu=2 \frac{\partial G_{0}}{\partial N} \text {, }
$$

where $A$ is defined by formula (1.6). Operator $A=A(k)$ is an entire function of $k$ and $A(k)$ is compact in $H=L^{2}(\Gamma)$ for any $k$ since $\Gamma$ is smooth. It is invertible for $\operatorname{Irn} k>0$. Hence, $(I+A(k))^{-1}$ is meromorphic and is defined on the whole complex plane $k$. Since $\partial G_{0} / \partial N$ for $x \notin \Gamma$ is an element of $H$ which is an entire function of $k$, one can see from (5), that $\mu=2(I+A(k))^{-1} \partial G_{0} / \partial N$ is meromorphic. From this argument and formula (4) it follows that $G(x, y, k)$ is meromorphic in $k$.

In $\S 1$ we emphasized that the location and properties of the complex poles of $G$ are of interest in applications. By the properties of the poles we mean mostly whether the poles are simple or not.

Proposition 1 The set of the poles of $G$ coincide with the set of the zeros of functions $\lambda_{n}(k), n=1,2,3 \ldots$, where $\lambda_{n}(k)$ are the eigenvalues of the operator $T(k)$ defined by formula (1.9).

Proof. Let $z$ be a pole of $G$,

$$
G=\frac{R(x, y)}{(k-z)^{r}}+\ldots
$$

From (4), (6), (2) after multiplying (4) by $(k-z)^{r}$ and taking $k=z$ we obtain

$$
\int_{\Gamma} G_{0}(s, t, z) \frac{\partial R(t, y)}{\partial N_{t}} \mathrm{~d} t=0, \quad s \in \Gamma .
$$

Since $R(x, y)$ is a degenerate kernel it follows from (7) that a function $f(t) \not \equiv 0$ exists such that

$$
\int_{\Gamma} G_{0}(s, t, z) f(t) \mathrm{d} t=0, \quad s \in \Gamma .
$$

It means that $\lambda_{n}(z)=0$ for some $n$.

Conversely, let equation (8) has a nontrivial solution. The function

$$
u(x)=\int_{\Gamma} G_{0}(x, t, z) f(t) \mathrm{d} t
$$

is a solution of the exterior Dirichlet problem

$$
\left(\triangle+z^{2}\right) u=0 \text { in } \Omega,\left.u\right|_{\Gamma}=0 \text {, }
$$

and

(11) $u$ satisfies the asymptotic condition at infinity.

If $z$ is not a pole of $G, \operatorname{Im} z \neq 0$, then $u \equiv 0$ in $\Omega$ and in $D$. It means that $f \equiv 0$ according to the jump relation. This is a contradiction. If $z$ is not a pole of $G$ and $\operatorname{Im} z=0$, then $u=0$ in $\Omega$ and $u \neq 0$ in $D$ only if $z^{2}$ is an eigenvalue of the interior Dirichlet problem for the Laplace operator. But such an eigenvalue is a (real) pole of $G(x, y, k)$. Again, we obtain a contradiction. This completes the proof. 
Remark 1. It is possible to find other functions whose zeros are poles of $G$ [12]. 2. Not much is known about the location of the complex poles of $G$ :

1) It is proved in [33], [19] that the complex poles $k_{j}$ of $G$ (only Dirichlet boundary condition was considered) satisfy the following inequality:

$$
\operatorname{Im} k_{j}<a-b \ln \left|k_{j}\right|, \quad b>0 \text {. }
$$

2) In [7] it was proved that a strip $-\varepsilon<\operatorname{Im} k<0, \varepsilon>0$ is free of the poles of the resolvent kernel of the Schrodinger operator with a finite potential $q(x) \in C^{1}$ for the exterior Dirichlet problem. This result shows that there exists a function $F(x)$ with the properties (1.23) such that the complex poles of the resolvent kernel of the Schródinger operator with $q(x) \in C_{0}^{1}$ satisfy inequality (1.22) for the exterior Dirichlet problem.

3) In [19] a study of the poles $k_{j}=\mathrm{i} \sigma_{j}, \sigma_{j}<0$ was carried out. It was proved that there exist infinitely many of such poles, and the number of poles with $\left|\sigma_{j}\right|<\sigma$ was estimated asymptotically for $\sigma \rightarrow \infty$.

4) The resolvent kernel of the Laplace operator of the exterior boundary value problem with the third boundary condition can have a pole $k=0$. In this case the solution of the corresponding nonstationary problem for the wave equation does not necessarily decay as $t \rightarrow \infty$. An example is given in [34] where the problem

$$
\begin{aligned}
& u_{t}=\Delta u \text { in } \Omega=\{|x| \geqslant R, t \geqslant 0\} \\
& u(x, 0)=0, u_{t}(x, 0)=f(r), \\
& \partial u / \partial r+R^{-1} u=0, \text { for } r=|x|=R, t \geqslant 0
\end{aligned}
$$

was considered. The solution can be found in the form

$$
u=\sum_{n, m} u_{n m}(r, t) \gamma_{n m}(w),
$$

where $\gamma_{n m}$ are the spherical harmonics. From the explicit formula for $u_{n m}$ it can be seen that $u_{00}(r, t)$ does not decay as $t \rightarrow \infty$ if $f(r) \geqslant 0$ and is finite. Another example is given in [32].

5) In [22] a criterion is given for an operator function $[I+A(k)]^{-1}$ to have only simple poles. If $z$ is a pole of this function, $I+A(k)=I+A(z)+$ $(k-z) A_{1}+\ldots$ then $z$ is a simple pole iff

$$
H=R(I+A(z))+A_{1} \operatorname{ker}\{I+A(z)\} .
$$

Unfortunately in order to apply this criterion in practice it is necessary to have such information about $I+A(z)$ and $A_{1}$, which is usually unavailable.

\section{§ 5 How to Calculate the Poles of the Green Function? Do the Poles Depend Continuously on the Boundary of the Obstacle?}

1. A general method for calculation the poles of Green functions in diffraction and scattering was given in [12], [13]. The poles coincide with the 
numbers $k_{j}$ for which $I+A(k)$ is not invertible (see equation (4.5)). Let $\left\{f_{j}\right\}$ be an orthonormal basis in $H=L^{2}(\Gamma)$ (or just a complete minimal system),

$$
\mu_{n}=\sum_{1}^{n} c_{j} f_{j}
$$

Substituting (1) in (4.5) and multiplying in $H$ by $f_{i}$ one obtains the system for unknown $c_{j}$ :

$$
\sum_{i=1}^{n} b_{i j}(k) c_{j}=0, b_{i j} \equiv\left([I+A(k)] f_{j}, f_{i}\right) \text {. }
$$

Here $(.,$.$) denotes the scalar product in H$. System (2) has a nontrivial solution iff

$$
\operatorname{det}\left[b_{i j}(k)\right]=0 \text {. }
$$

The left-hand side of this equation is an entire function of $k$. Let $k_{m}^{(n)}, m=1,2$, $3, \ldots$ be its roots. In [13] the following proposition is proved.

Proposition 1 The limits $\lim _{n \rightarrow \infty} k_{m}^{(n)}=k_{m}$ exist and are the poles of the Green function $G(x, y, k)$ of the exterior Dirichlet problem. Every pole of $G(x, y, k)$ can be obtained in such a way.

Remark 1. The same approach is valid for various boundary conditions (Neumann and third boundary conditions included), and for the potential scattering by a finite potential [12].

Remark 2. This approach is a variant of the general projection method.

Sketch of the proof. First we show that $k_{m}^{(n)} \rightarrow k_{m}$ as $n \rightarrow \infty$. In the complex plane we choose a circle $K_{R}$ of arbitrary radius $R$. Suppose that the points $k_{1}, \ldots$, $k_{s}$ for which $I+A(k)$ is not invertible lie inside $K_{R}$ and the remaining points $k_{m}$ lie outside $K_{R}$. Denote by $\varepsilon>0$ a small number, by $D_{\varepsilon, R}=\left\{k:\left|k-k_{j}\right| \geqslant \varepsilon\right.$, $|k| \leqslant R\}$. We assume that the circles $\left|k-k_{j}\right| \leqslant \varepsilon, 1 \leqslant j \leqslant s$ do not overlap. The operator $[I+A(k)]^{-1}$ is uniformly bounded on $D_{\varepsilon, R}$ :

$$
\left\|[I+A(k)]^{-1}\right\| \leqslant M, \quad k \in D_{\varepsilon, R}, M=M_{\varepsilon, R} .
$$

Equation (2) can be written as

$$
\mu_{n}+P_{n} A(k) \mu_{n}=0 \text {, }
$$

where $P_{n}$ is the projector on the span of $f_{1}, \ldots, f_{n}$. Since $P_{n} \rightarrow I$, where $\rightarrow$ denotes strong convergence of the operators on $H$, and $A(k)$ is compact, we conclude that $\left\|A(k)-P_{n} A(k)\right\| \rightarrow 0$ as $n \rightarrow \infty$. Therefore $\| I+A(k)-$ $\left[I+P_{n} A(k)\right] \| \rightarrow 0$ as $n \rightarrow \infty$. It means that for $n$ sufficiently large operators $I+P_{n} A(k)$ are invertible in $D_{\varepsilon, R}$, because $I+A(k)$ is invertible in $D_{\varepsilon, R}$. Therefore all roots of equation (3) for $n$ sufficiently large lie in the union of the circles

$$
\left|k-k_{j}\right| \leqslant \varepsilon, \quad|k| \leqslant R .
$$

Since $\varepsilon>0$ is arbitrarily small, this means that uniformly in the domain $|k| \leqslant R$ the limits exist: 


$$
\lim _{n \rightarrow \infty} k_{j}^{(n)}=k_{j} \text {. }
$$

Conversely, let $k_{j},\left|k_{j}\right|<R$ be an arbitrary pole of $G(x, y, k)$. Then operator $I+A\left(k_{j}\right)$ is not invertible. Suppose that in the circle $\left|k_{j}-k\right|<\varepsilon$ there are no numbers $k_{m}^{(n)}$ and no points $k_{i}$ for $\mathrm{i} \neq j$. Then $\left\|[I+A(k)]^{-1}\right\| \leqslant M$ for $\left|k-k_{j}\right|=$ $\varepsilon$ and for $n$ sufficiently large $\left\|\left[I+P_{n} A(k)\right]^{-1}\right\| \leqslant M_{1}$. Since there are no numbers $k_{m}^{(n)}$ inside the circles $\left|k-k_{j}\right|<\varepsilon$, the operator $I+P_{n} A(k)$ is invertible for $\mid k-$ $k_{j} \mid \leqslant \varepsilon$ and $\left[I+P_{n} A(k)\right]^{-1}$ is an analytic operator function for $\left|k-k_{j}\right| \leqslant \varepsilon$. From the maximum modulus principle we obtain a uniform (with respect to $n$ ) estimate $\left\|\left[I+P_{n} A(k)\right]^{-1}\right\| \leqslant M_{1}$ for $\left|k-k_{j}\right| \leqslant \varepsilon$. But from this estimate we conclude that the operator $[I+A(k)]^{-1}$ exists for $\left|k-k_{j}\right| \leqslant \varepsilon$, which is a contradiction. This completes the proof.

Remark 3. The method gives a uniform approximation to the complex poles in any compact domain of the complex plane $k$.

2. In this section we show that in any compact domain of the complex plane the complex poles depend continuously on the boundary in the following sense. Consider a parametrized equation of the boundary $\Gamma$

$$
x_{j}=x_{j}\left(t_{1}, t_{2}\right), \quad 1 \leqslant j \leqslant 3,0 \leqslant t_{1}, t_{2} \leqslant 1
$$

where $x_{j} \in C^{2}$.

$$
\text { Assume that a boundary } \Gamma_{\varepsilon} \text { obeys the following equation }
$$

$$
x_{j}(\varepsilon)=x_{j}\left(t_{1}, t_{2}\right)+\varepsilon y_{j}\left(t_{1}, t_{2}\right), \quad 1 \leqslant j \leqslant 3 .
$$

Where $y_{j} \in C^{2}$. Let $G\left(G_{\varepsilon}\right)$ be the Green function of the exterior Dirichlet problem in $\Omega, \partial \Omega=\Gamma\left(\Omega_{\varepsilon}, \partial \Omega_{\varepsilon}=\Gamma_{\varepsilon}\right)$. Let $k_{j}\left(k_{j}(\varepsilon)\right)$ be the poles of $G\left(G_{\varepsilon}\right)$.

Proposition 2 If $\varepsilon \rightarrow 0$ then $k_{j}(\varepsilon) \rightarrow k_{j}$ uniformly for $\left|k_{j}\right| \leqslant R$, where $R>0$ is an arbitrary large fixed number.

Proof. Denote by $\Delta=\left\{0 \leqslant t_{1}, t_{2} \leqslant 1\right\}$. Then $k_{j},\left|k_{j}\right| \leqslant R$ are the points of the complex plane $k$ at which the operator $I+A(\mathrm{k})$ defined by formula (4.5) is not invertible. Operator $I+A(k, \varepsilon)$ is not invertible at the points $k_{j}(\varepsilon)$. Here the operator $A(k, \varepsilon)$ is the counterpart of $A(k)$ for $\Gamma_{\varepsilon}$. Both operators can be written in the form

$$
A(k, \varepsilon)=\int_{\Delta} \frac{\partial G_{0}}{\partial N} \mu J(t, \varepsilon) \mathrm{d} t_{1} \mathrm{~d} t_{2}
$$

where $J(t, \varepsilon) \mathrm{d} t_{1} \mathrm{~d} t_{2}$ is the element $\mathrm{d} t$ of the area of $\Gamma_{\varepsilon}$; for $\varepsilon=0$ we obtain the operator $A(k)$. Since $x_{j}, y_{j} \in C^{2}$ the function $J(t, \varepsilon)$ is continuous (actually $J(t, \varepsilon)$ $\in C^{1}$ )

$$
\lim J(t, \varepsilon)=J(t) \text { as } \varepsilon \rightarrow 0 .
$$

Thus,

$$
\|A(k, \varepsilon)-A(k)\| \rightarrow 0 \text { as } \varepsilon \rightarrow 0,|k| \leqslant R .
$$


Now we can use the arguments given in the proof of Proposition 1. The role of $n$ is played by $\varepsilon$. Consider the union $K_{\varepsilon}$ of the circles $\left|k-k_{j}\right| \leqslant \delta$, where $\delta>0$ is an arbitrary small fixed number, $\left|k_{j}\right|<R, 1 \leqslant j \leqslant s$ and the circles do not overlap. By $D_{R, \delta}$ we denote $K_{R} \backslash K_{\delta}, K_{R}=\{k:|k| \leqslant R\}$.

In $D_{R, \delta}$ operator $I+A(k)$ is invertible. Because of condition (12) for $\varepsilon$ sufficiently small the operator $I+A(k, \varepsilon)$ is also invertible in $D_{R, \delta}$. This means that $k_{j}(\varepsilon) \in K_{\delta}$ for an $\varepsilon$ sufficiently small. Since $\delta>0$ is arbitrarily small the proof of Proposition 2 is complete.

Remark 4. It is possible to estimate $k_{j}(\varepsilon)-k_{j}$. In a general setting this type of perturbation theory was studied in [40], [41].

\section{Appendix 1. Losses in Open Resonators [14]}

Diffraction losses for the $n$-th mode in an open confocal resonators can be calculated by the formula

$$
\alpha_{n}=1-\left|\lambda_{n}\right|^{2}, \quad n=0,1,2 \ldots,
$$

where $\lambda_{n}$ are the eigenvalues of the following operator

$$
A f=\lambda f, \quad A f=\frac{b}{2 \pi} \int_{s} \exp \{-\mathrm{i} b(x, u)\} f(u) \mathrm{d} u,
$$

and $S \subset \mathbf{R}^{2}$ is a central-symmetric domain, $b>0$. It is easy verify that $A$ is normal. Thus $\left|\lambda_{n}\right|=s_{n}$, where $s_{n}=\lambda_{n}\left\{\left(A^{*} A\right)^{1 / 2}\right\}$ are the $s$-numbers of $A$. From the result, given in [36] it follows, that

$$
s_{n}\left(S_{1}\right) \leqslant s_{n}\left(S_{2}\right) \text { if } S_{1} \subset S_{2} \text {. }
$$

From this we obtain the following inequalities

$$
\alpha_{n e} \leqslant \alpha_{n} \leqslant \alpha_{n i}, \quad n=0,1,2, \ldots,
$$

where $\alpha_{n e}\left(\alpha_{n i}\right)$ are the losses for the mirrors $S_{e}\left(S_{i}\right), S_{e} \supset S \supset S_{i}$. In [14] the following formula was also obtained:

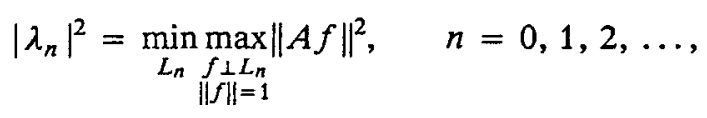

where $L_{n}$ is a $n$-dimensional subspace of $H=L^{2}(S)$. The following conjecture was discussed in [14]: among all central-symmetric mirrors $S$ with a fixed area $|S|$ the circle has minimal diffraction losses.

\section{Appendix 2. An Example on Complex Scaling}

In connection with spectral properties of the Schrödinger operator recently the complex scaling technique has attracted much attention [16]. The main idea is to consider solutions of the Schrödinger equation for complex values of $r=|x|$. 
This idea was used by the author as early as 1963 in order to prove the absense of positive discrete spectrum of the Laplace operator of the Dirichlet problem in some infinite domains with infinite boundaries [15]. The arguments given in [15] are not elementary. Here we use the same idea as in [15] and give a very simple proof of the following (known) proposition.

Proposition 1 Let $D \subset \mathbf{R}^{3}$ be a bounded domain with a smooth closed connected boundary $\Gamma, \Omega=\mathbf{R}^{3} \mathcal{D}$,

(1) $\left(\Delta+k^{2}\right) u=0 \quad$ in $\Omega, k^{2}>0$,

(2) $u \in L^{2}(\Omega)$,

(3) $\left.u\right|_{r}=0$.

Then $u(x) \equiv 0$ in $\Omega$.

Proof. By the Green formula we have

$$
u(x)=-\int_{\Gamma} g^{+} \mu \mathrm{d} t, \quad \mu=\frac{\partial u}{\partial N}, \quad g^{+}=\frac{\exp \left(\mathrm{i} k r_{x y}\right)}{4 \pi r_{x y}} .
$$

(From (2) it follows, that $\nabla u \in L^{2}(\Omega)$ and hence a sequence $r_{n} \rightarrow \infty$ exists such that

$$
\int_{|x|=r_{n}}\left\{|u|^{2}+|\partial u / \partial N|^{2}\right\} \mathrm{d} s \rightarrow 0 \text { as } n \rightarrow \infty .
$$

Therefore the integral over the large sphere in the Green formula tends to zero.) Let $x=r w$, where $w$ is a unit vector, and let $z=r \exp (\mathrm{i} \theta)$. The function $u(x)=$ $u(r w)$ is considered as a function of the complex variable $z$. Since

$$
g^{+}=\exp \frac{\left\{\mathrm{i} k \sqrt{r^{2}-2 r|t| \cos \alpha+|t|^{2}}\right\}}{\sqrt{r^{2}-2|t| r \cos \alpha+|t|^{2}}}, \quad \alpha=\hat{w} t \text {, }
$$

it is clear, that $G_{0}$ is analytic in $z=r \exp (\mathrm{i} \theta)$ for $|z| \geqslant R$, where $R$ is sufficiently large, such that if $r>R$ then the inequality holds:

$$
r^{2}>2 r d+d^{2}, \quad d=\max _{t \in \Gamma}|t| \text {. }
$$

Thus for $|z|>R$ the function $\sqrt{z^{2}-2 z|t| \cos \alpha+|t|^{2}}$ is analytic if we fix some branch of the radical. From (3) it follows that

$$
u=\frac{\exp (\mathrm{i} k z)}{z} f_{1}(z),
$$

where $f_{1}$ is analytic in $|z|>R$ and

$$
f_{1}=0(1) \text { for }|z|>r \text {. }
$$

Exactly the same arguments lead to the formulas:

$$
u=-\int_{\Gamma} g^{-} \mu \mathrm{d} t, \quad g^{-}=\frac{\exp \left(-\mathrm{i} k r_{x y}\right)}{4 \pi r_{x y}},
$$


(11) $u=\frac{\exp (-\mathrm{i} k z)}{z} f_{2}(z)$,

where $f_{2}(z)$ is analytic in $|z|>R$ and

$$
f_{2}=0(1) \text { for }|z|>R \text {. }
$$

Hence

$$
u(z)=\frac{\mathrm{e}^{\mathrm{i} k z}}{z} f_{1}=\frac{\mathrm{e}^{-\mathrm{i} k z}}{z} f_{2}(z) \text { for }|z|>R .
$$

Formula (12) is contradictory unless $u \equiv 0$. To prove that we use a known uniqueness lemma for analytic functions (see also Bibliographical notes).

Lemma Let $D$ be a domain on the complex plane $z, C$ be its boundary. Let $D$ contain the half plane $\operatorname{Re} z>a$. Let $f(z)$ be analytic in $D$, continuous in $D+C$ and

$$
\ln |f(z)| \leqslant A|z| \text { for }|z|>R, z \in D,
$$

where $A=$ const $>0$, and $R$ is an arbitrary large fixed number,

$$
\ln |f(z)| \leqslant-h(|z|), \quad z \in C,
$$

where $h(t)>0$ is a continuous function such that

$$
\int_{1}^{\infty} t^{-2} h(t) \mathrm{d} t=\infty
$$

Then $f(z) \equiv 0$ in $D$.

In our case $f(z)=u(z), D$ can be chosen so that $C$ coincides outside of some large circle with the rays $\arg z=3 \pi / 4, \arg z=5 \pi / 4, h(t)=$ const $+\frac{t}{\sqrt{2}}$,
so that (16) is satisfied. We have

$$
\ln |u(z)| \leqslant k|z|-\ln |z|+\ln \left|f_{1}\right| \leqslant A|z|, \quad z \in D,
$$

(since $\left|f_{1}\right| \leqslant C_{1}$ we have $\ln \left|f_{1}\right| \leqslant C_{2}$ ).

$$
\ln |u(z)| \leqslant-\frac{k|z|}{\sqrt{2}}+\text { const, for }|z|>R, z=|z| \exp (\mathrm{i} 3 \pi / 4) .
$$

Similar estimate holds on the ray $\arg z=5 \pi / 4$. From the preceding lemma it follows that $u(z) \equiv 0$. Thus. $u(r, w)=0$ for $r>R$. By the unique continuation theorem we conclude that $u \equiv 0$ in $\Omega$.

\section{Appendix 3. Variational Principles for Eigenvalues of Compact Nonselfadjoint Operators}

Let $T$ be a linear compact operator on a Hilbert space $H, \lambda_{j}$ be its eigenvalues, $\left|\lambda_{1}\right| \geqslant\left|\lambda_{2}\right| \geqslant \ldots, r_{j}\left(t_{j}\right)$ be the moduli of the real (imaginary) parts of the eigenvalues, $r_{1} \geqslant r_{2} \geqslant \ldots\left(t_{1} \geqslant t_{2} \geqslant \ldots\right)$. Let $L_{j}, M_{j}, N_{j}$ be the eigenspaces 
of $T$ corresponding to $\lambda_{j}, r_{j}, t_{j}$ respectively. Note that $r_{j}$ is not necessarily equal to $\left|\operatorname{Re} \lambda_{j}\right|$. We can set a one to one correspondence between $r_{j}$ and $\left|\operatorname{Re} \lambda_{j}\right|$, and $M_{j}$ and $L_{j}$, putting $M_{i}=L_{j(i)}$ where $j(i)$ is so chosen, that $\left|\operatorname{Re} \lambda_{j(i)}\right|=r_{i}$. The same is true for $L_{j}$ and $N_{j}$. Let $\tilde{L}_{j}=\sum_{k=1}^{j}+L_{k}$ and $\tilde{M}_{j}, \tilde{N}_{j}$ are defined similarly, $\dot{+}$ means the direct sum, $\tilde{L}_{j}{ }^{1}$ means the direct complement to $\tilde{L}_{j}$ in $H$.

Theorem The following formulas hold:

$$
\begin{aligned}
& \left|\lambda_{j}\right|=\max _{x \in \bar{L}_{j-1}^{1}} \min _{\substack{y \in H \\
(x, y)=1}}|(T x, y)|, \\
& r_{j}=\max _{x \in \bar{M}_{j-1}^{1}} \min _{\substack{y \in H \\
(x, y)=1}}|\operatorname{Re}(T x, y)|, \\
& t_{j}=\max _{x \in \bar{N}_{j-1}^{1}} \min _{\substack{y \in H \\
(x, y)=1}}|\operatorname{Im}(T x, y)| .
\end{aligned}
$$

Here $(x, y)$ denotes the scalar product in $H$.

Proof. We prove formula (1) for $j=1$. The proof of other statements of the Theorem are similar. For $j=1$ formula (1) can be written as

$$
\lambda_{1}=\max _{x \in H} \min _{\substack{y \in H \\(x, y)=1}}|(T x, y)| \text {. }
$$

For a fixed $x$ we write $T x=\lambda x+z$, where $z \in x^{\perp}, x^{\perp}$ is the subspace of all vectors orthogonal to $x$ and $\lambda$ is a number. Thus $(T x, y)=\lambda+(z, y)$. Let us represent $y$ in the form $y=\mu x+u, u \in x^{\perp}$. From the condition $(x, y)=1$ it follows that $\mu=|x|^{-2}$. Thus $(T x, y)=\lambda+(z, u)$. We have

$$
\min _{\substack{y \in H \\
(x, y)=1}}|(T x, y)|=\min _{u \in x^{\perp}}|\lambda+(z, u)|=\left\{\begin{array}{cc}
|\lambda| & \text { if } z=0, \\
0 & \text { if } z \neq 0 .
\end{array}\right.
$$

Formula (5) implies (4).

If $j>1$ then $\max _{x \in \bar{L}_{j-1}^{1}} \min _{\substack{y \in H \\(x, y)=1}}=\max _{\substack{x \in \bar{L}_{j-1}^{1} \\ T x=\lambda x}}|\lambda|=\left|\lambda_{j}\right|$. Formulas (2), (3) are treated simi-
larly.

Remark 1. If $T_{n}$ is compact and $\left\|T-T_{n}\right\| \rightarrow 0$ as $n \rightarrow \infty$, then $\lambda_{j}\left(T_{n}\right) \rightarrow \lambda_{j}(T)$, $\forall j$. This fact permits an approximate calculation of the spectrum of $T$ using in (1) - (3) the operator $T_{n}$ instead of $T$. In particular one can take $n$-dimensional operator $T_{n}$ (dimrange $T_{n}=n$ ).

\section{Bibliographical Notes}

Section 1. Questions discussed here are of interest for engineers and physicists [2] - [4], [32]. They attracted considerable attention of mathematicians in recent years [1], [19], [3], Appendix. Our knowledge of the spectral structure of nonselfadjoint operators is very limited. For example, it is not known how to investigate this structure of the equation (1.12). If a nonselfadjoint operator is a 
weak perturbation (in the sense defined in section 2) of a selfadjoint operator some information is available (see A. Marcus [17], V. Kacnelson [18], M. Agranovich [3]). For dissipative operators there exist some theorems about completeness of their root systems [1], [11]. No answer to question 5) is known.

Section 2. Properties of the bases of a Hilbert space are described in [1] in the form convenient for our purpose. A rigorous study of the spectral properties of the integral operators arising in diffraction theory was initiated in [12], [11], [13]. Questions put forward by B. Kacenelenbaum were stimulating for these studies. M. S. Agranovich [3] has made further contribution to this theory. Essential to his results were the results due to A. Markus [17] and V. Kacnelson [18]. The theory of pseudo-differential operators is now well developed. A summary of main results of this theory is given in [20], [21], [3], [38]. On the basis property without brackets see [43].

M. S. Agranovich [3] applied the theory of pseudo-differential operators to the integral equations of diffraction theory.

Section 3. References are given in the section.

Section 4. The questions discussed here are of interest in applications. Proposition 1 was proved in [29]. A part of it was proved in [12]. The scheme for the study of analytic continuation of the resolvent kernel of the Schrödinger operator was given in [5], [6], [25], [26]. Analytic properties of the scattering matrix for acoustic wave scattering by an obstacle was studied in [39]. Eigenfunction expansion theorems for nonselfadjoint Schrödinger operator are proved in [8], [9] and the properties of the resolvent in the complex plane of the spectral parameter $k$ were used in the proofs. In [19] a study of the purely imaginary poles of the Green function of the exterior Dirichlet and the Neumann problem is given. The known criteria for a pole of an operator-valued function to be simple, including criterion (4.17) unfortunately are difficult to apply: so far no applications of these criteria appear to be known.

In [27] it is proved that for the complex poles of the Green function of the exterior Neumann problem for a convex domain in $\mathbf{R}^{3}$ with a smooth boundary which has a positive Gaussian curvature, the function $F(x)$ in formula (4.22) can be taken as $F(x)=\varepsilon|x|^{1 / 3}$, for some small $\varepsilon>0$. In [28] it was shown how to pose correctly the problem of finding root vectors corresponding to the complex poles of the Green functions.

In [42] the analytic continuation of the resolvent of some general differential operators is studied.

There is an example in [37] which shows that a root system of a nonselfadjoint operator may form a basis of $H$, but some other root system of the same operator may not form a basis of $H$.

In the literature the radiation condition in the form

$$
u-\frac{\exp (\mathrm{i} k r)}{r}\left(1+o\left(\frac{1}{r}\right)\right) \text { as } r \rightarrow \infty
$$

is often used for $\operatorname{Im} k<0$, i.e. for exponentially increasing solutions of the problem (4.10). It is assumed in such cases that the solution of the boundary 
value problem satisfying the radiation condition in unique. This is false. A simple example is the function $u=g^{+} * f-g^{-} * f$, where $g^{+}, g^{-1}$ are defined by formulas (3), (9) of Appendix 2, $f \in C_{0}^{\infty}$ is arbitrary, * denotes convolution. It is clear that $\left(\Delta+k^{2}\right) u=0$ in $\mathbf{R}^{3}$ and $u$ satisfies the radiation condition for $\operatorname{Im} k<$ 0 , but $u \neq 0$. The right asymptotic condition for exponentially increasing solutions is given in [28], where it is proved that for $\operatorname{Im} z<0$ the solution of the problem (4.10) has, in a neighborhood of infinity the following form $u=r^{-1} \exp (\mathrm{i} z r) \sum_{j=0}^{\infty} f_{j}(\alpha) r^{-j}, r=|x|, \alpha=x|x|^{-1}$, and the series converges absolutely and uniformly for sufficiently large $r$.

Section 5. The simple method for calculation of the complex poles is given in [12], [13]. It is essentially a variant of the projection method and the arguments show that the complex poles depend continuously on the boundary. The same arguments prove the continuous dependence of these poles on the parameters if the kernel depends continuously on these parameters.

The results of Appendix 3 was proved in [29]. In [30], [31] it was shown rigorously that the solution of the exterior Dirichlet boundary value problem is the limit of the solutions of the potential scattering problem when the potential goes to infinity in $D$ and is equal to 0 in $\Omega$. Here as usually $D=\mathrm{R}^{3} \backslash \Omega, \Omega$ is the exterior domain. In [10] [35] behavior as $t \rightarrow \infty$ of the solution of the wave equation in exterior domain was studied in case when the resolvent kernel of the corresponding stationary problem cannot be analytically continued through the continuous spectrum.

It is possible to conclude from formula (12) in Appendix 2 that $u(z) \equiv 0$ without making use of Lemma of this Appendix. Indeed, since $f_{1}, f_{2}$ are analytic and bounded in some neighborhood of infinity they behave asymptotically as $C_{n} z^{-n}, n \geqslant 0$. If $z=\mathrm{i} y$ in formula (12) of Appendix 2 and $y \rightarrow+\infty$, then the left-hand side of this formula goes to zero, while the right-hand side goes to infinity unless $f_{1}=f_{2}=0$. This simple argument was pointed out by B. A. Taylor. In [15], where the boundary of the domain was infinite it was necessary to use Lemma from Appendix 2. It is interesting to mention that exactly the same arguments prove the following proposition.

Proposition 1 Let $u$ be a solution of the problem (1)-(2) of Appendix 2. Then $u \equiv 0$.

Note that no assumptions about boundary values of $u$ are made in this proposition.

\section{Unsolved Problems}

1. To what extent do the complex poles of the Green function determine the obstacle?

2. It is true that the complex pole of the Green function for the exterior Dirichlet problem are simple?

3. Does the order of a complex pole coincide with the order of zero of the corresponding eigenvalue? (see Proposition 1 in $\S 4$ ). 


\section{References}

[1] Gohberg, I.; Krein, M.: Introduction to the theory of linear nonselfadjoint operators. Amer. Math. Soc. 1969. = Transl. of Math. monographs, Vol. 18

[2] Dolph, C. L.; Scott, R. A.: Recent developments in the use of complex singularities in electromagnetic theory and elastic wave propagation. In: Electromagnetic scattering. New YorkLondon: Academic Press 1978, 503-570

[3] Voitovich, N.; Kacenelenbaum, B.; Sivov, A.: Generalized method of eigenoscillations in diffraction theory (with Appendix written by M. S. Agranovich) Moscow: Nauka 1977 (in Russian)

[4] Baum, C. E.: Emerging technology for transient and broad-bond analysis and synthesis of antennas and scatterers. Proc. IEEE 64 (1976) 1598- 1676

[5] Ramm, A. G.: Analytic continuation of solutions of the Schrödinger equation in spectral parameter and behavior of solutions of nonstationary problem as $t \rightarrow+\infty$. Uspehi Mat. Nauk. 19 (1964) $192-194$

[6] Ramm, A. G.: Some theorems on analytic continuation of the Schrödinger operator resolvent kernel in spectral parameter. Izvestja Acad. Nauk Armjan. SSR, Mathematics 3 (1968) $443-464$; MR $42 \# 5563$

[7] Ramm, A. G.: The exponential decrease of a solution of a hyperbolic equation. Diff. eq. 6 (1970) 1598 - 1599; MR 44 \#631

[8] Ramm, A. G.: Eigenfunction expansion for nonselfadjoint Schrődinger operator. Sov. phys.Doklady 15 (1970) 231 - 234; MR $42 \# 703$

[9] Ramm, A. G.: Expansions in eigenfunctions of an exterior boundary-value problem for a nonselfadjoint differential operator. Diff. eq. 7 (1971) 565-569; MR 44 \#2094

[10] R. a mm, A. G.: On the limiting amplitude principle. Diff. eq. 4 (1968) 370-373; MR 37 \#1759

[11] Ramm, A. G.: Eigenfunction expansion of a discrete spectrum in diffraction problems. Radio Eng. Electr. Phys. 18 (1973) 364-369; MR 50 \#1641

[12] Ramm, A. G.: Exterior problems of diffraction. Radio Eng. Electr. Phys. 17 (1972) 1064 - 1067; MR 51 \#864

[13] Ramm, A. G.: Computation of quasistationary states in nonrelativistic quantum mechanics. Sov. Phys. Doklady 17 (1972) $522-524$, MR 56 \#14326

[14] Ramm, A. G.: Diffraction losses in open confocal cavities with mirrors of arbitrary shapes. Opt. Spectrosc. 40 (1976) $89-90$

[15] Ramm. A. G.: About the absence of the discrete positive spectrum of the Laplace operator in some infinite domains. Vestnik Leningrad. Gosud. Univ., ser. atron., math., mech. 13 (1964) 153 - 156, NI (1966) 176; MR 30\#1295

[16] Complex scaling in the spectral theory of the Hamiltonian. Int. J. of Quantum Chemistry $14 \mathrm{NY}$ (1978)

[17] Marcus, A. S.: The root vector expansion of a weakly perturbed selfadjoint operator. Sov. math. Doklady 3 (1962)

[18] Kacnelson, V.: Conditions for a system of root vectors of some classes of nonselfadjoint operators to form a basis. Funct. anal. and applic. 1 (1967) $39-51$ (Engl. transl. pp. 122 - 132)

[19] Lax, P. D.; Phillips, R. S.: Decaying modes for the wave equation in the exterior of an obstacle. Comm. Pure Appl. Math. 22 (1969) 737 - 787

[20] Agmon, S.: Lectures on elliptic boundary value problems. New York: Van Nostrand 1965

[21] Seeley, R.: Refinement of the functional calculus of Calderon and Zygmund. Proc. Konikl. Nederl. Acad. ser A 68 (1965) 521-531

[22] Howland, J.: Simple poles of operator-valued functions. J. Math. Anal. Appl. 6 (1971) 12 - 20

[23] Ramm, A. G.: A remark on the theory of integral equations. Diff. eq. 8 (1972) 1177-1180; MR 47 \#2284

[24] Decuard, D.; Foias, C.; Pearcy, C.: Compact operators with root vectors that span. Proc. Amer. Math. Soc. 76 (1979) $101-106$

[25] Ramm, A. G.: On diffraction problems in domains with infinite boundaries. Proc. 3-rd allunion symposium on wave diffraction. Moscow: Nauka 1964 28-31 
[26] Ramm, A. G.: Analytic continuation of resolvent kernel of the Schrodinger operator in spectral parameter and the limiting amplitude principle. Doklady Acad. Nauk. Azerb. SSR 21 (1965) $3-7$

[27] Babich, V. M.; Grigorjeva, N. S.: Asymptotic properties of solutions to some three dimensional wave problems. J. Sov. Math. 11 (1979) $372-412$

[28] Vainberg, B. R.: On eigenfunctions of an operator corresponding to the poles of the analytic continuation of the resolvent through the continuous spectrum. Math USSR Sborn. 16 (1972) $307-322$

[29] Ramm, A. G.: Theory and applications of some new classes of integral equations. New York: Springer 1980

[30] Ramm, A. G.: A method for solution of the Dirichlet problem in infinite domains. Itvestija vusov, mathematics 5 (1965) 124-127; MR 32 \#7993

[31] Ramm, A. G.: Asymptotic behaviour of the eigenvalues and eigenfunction expansions for the Schrodinger operator with increasing potential in domains with infinite boundaries. Izvestija Acad. Nauk, Armjan. SSR; Mathematics 4 (1969) 442-467; MR 42 \#3457

[32] Dolph, C. L.: The integral equation method in scattering theory. Problems in analysis. Princeton, N. J.: Princeton Univ. Press 1970, pp. $201-227$

[33] Lax, P. D.; Phillips, R. S.: A logarithmic bound on the location of the poles of the scattering matrix. Arch. Rat. Mech. Anal. 40 (1971) $268-280$

[34] Asakura, F.: On the Green function for $\Delta-\lambda^{2}$ with the third boundary condition in the exterior domain of a bounded obstacle. J. Math. Kyoto Univ. 18-3 (1978) 615-625

[35] Ramm, A. G.: Necessary and sufficient conditions for the validity of the limiting amplitude principle. Izvestija vusov, Mathematics, N5 (1978) $96-102$ (Engl. transl pp. 71-76)

[36] Ramm, A. G.: Discrimination of random fields in noises, Problems of Inform. Transmission 9 (1973) 192-205; MR 48 \#13439

[37] Ilin, V. A.: Existence of the reduced root system of a nonselfadjoint ordinary differential operator. Trudy math. inst. Steklova 142 (1976) $148-155$ (in Russian)

[38] Shubin, M. A.: Pseudo-differential operators and the spectral theory. Moscow: Nauka 1978 (in Russian)

[39] Lax, P. D.; Phillips, R. S.: Scattering theory. New York-London: Academic Press 1967

[40] Vainberg, M. M.; Trenogin, V.: Bifurcation theory of solutions of nonlinear equations. Leiden: Noordhoff 1974

[41] Kat o, T.: Perturbation theory for linear operators. Berlin-Heidelberg-New York: Springer 1966

[42] Vainberg, B. R.: On the analytic properties of the resolvent for a class of operator pencils. Math. USSR Sborn. 6 (1968) $241-272$

[43] Ramm, A. G.: On the basis property for the root vectors of some nonselfadjoint operators. J. Math. Anal. Appl. (to appear)

Prof. A. G. Ramm

Dept. of Mathematics

University of Michigan

347 West Engineering Building

Ann Arbor, MI 48 109, USA

(Received February 22, 1980) 